\title{
Neurology
}

\section{Nursing the seizure patient}

\section{Charlene Mallott}

The role of the registered veterinary nurse in the care and management of patients suffering with seizures is important. For a veterinary nurse to be able to provide the best possible care, prompt and accurate recognition of the seizure and the different presentations is essential.

Seizures can present as generalized or focal. Generalized seizures typically present as tonic/clonic manifesting as loss of consciousness, hypertonia, collapse and jerking of limbs, but can also be clonic, myoclonic, atonic or absence. Focal seizures commonly manifest as facial twitching or a behavioural disorder such as vacancy, fly catching or unusual aggression. During a seizure, loss of bladder or bowel control may occur and breathing may be compromised causing cyanosis.

Seizures can be self-limiting, such as an isolated event. Cluster seizures is classified as two or more seizures in 24 hours. Reflex seizure is caused by a recognized stimulant, toxic or metabolic insult. Status epilepticus (SE) is continuous seizure activity or failure to return to normality between seizures.

Emergency treatment must be administered to a seizuring patient and to assist with this a patent intravenous catheter is recommended. Initial administration of anticonvulsants, typically benzodiazepines (or leviteracetam in some cases, such as liver pathology) aims to stop seizure activity and allow stabilization of the patient's vital parameters.

Monitoring of heart rate, respiratory rate and effort, blood pressure and temperature is vital and, if necessary, the modified Glasgow coma score must be obtained and monitored for improvement or deterioration in a severe SE or comatose patient

Investigation of the underlying cause of the seizure(s) can commence once the clinician in charge feels the patient is stable enough. A thorough neurological assessment will take place, followed by blood tests, with possible magnetic resonance imaging, electroencephalograph and spinal fluid analysis
There are many conditions that cause seizures but the most commonly seen conditions are idiopathic epilepsy, inflammatory processes such as infectious or immune-mediated diseases, toxic or metabolic insult, neoplasia, trauma or anatomical abnormality.

Education and advice can be given to owners to assist in patient care at home and offer reassurance. It is helpful to clarify with owners doses of any anti-epileptic drugs (AED) and to provide directions for rectal diazepam should this be prescribed. Owners should be made aware of potential side effects of prescribed medication. It is good practice to advise the owner to keep a written diary of any seizure activity the patient has at home to help keep a clear history.

\section{KEY LEARNING OBJECTIVES}

- Recognize the different types of seizures

- Understand the importance of recognizing a seizure and the emergency treatment that should be initiated

- Understand nursing considerations when caring for a patient with seizures

\section{MULTIPLE CHOICE QUESTIONS}

1. Which of the following is NOT a typical presentation of a generalized seizure?
(A) Fly catching
(B) Collapse
(C) Hypertonia
(D) Loss of bladder control

2. Which of the following is advised to assist with emergency treatment of a patient having a seizure?
(A) Endotracheal tube placement
(B) Intravenous catheter placement
(C) Indwelling urinary catheter placement
(D) Phlebotomy for blood tests

3. Which of these is NOT a commonly seen side effect of AED?
(A) Polydipsia
(C) Alopecia
(B) Polyphagia
(D) Ataxia

\section{Nursing the vestibular patient}

\section{Charlene Mallott}

Vestibular patients can present in a number of different ways and in some cases it can be difficult to distinguish a vestibular episode from that of a seizure. It greatly benefits the registered veterinary nurse to be able to recognize a vestibular episode to allow better suited nursing care. Patients suffering from vestibular syndrome can present with one or all of the following symptoms:

- Head tilt

- Nystagmus

- Strabismus

- Ataxia

- Circling or falling to one side

- Wide-based stance

- Severe cases of vestibular disease can result in a patient 'crocodile rolling' in one continuous direction due to severe imbalance. 